\title{
Results of a Multicenter, Randomized, Double-Masked, Placebo-Controlled Clinical Study of the Efficacy and Safety of Visomitin Eye Drops in Patients with Dry Eye Syndrome
}

Vladimir V. Brzheskiy · Elena L. Efimova · Tatiana N. Vorontsova • Vladimir N. Alekseev •

Olga G. Gusarevich • Ksenia N. Shaidurova · Alla A. Ryabtseva • Olga M. Andryukhina · Tatiana G. Kamenskikh •

Elena S. Sumarokova · Eugeny S. Miljudin · Eugeny A. Egorov • Oleg I. Lebedev · Alexander V. Surov •

Andrii R. Korol · Illia O. Nasinnyk · Pavel A. Bezditko · Olena P. Muzhychuk · Vladimir A. Vygodin ·

Elena V. Yani · Alla Y. Savchenko · Elena M. Karger · Oleg N. Fedorkin · Alexander N. Mironov ·

Victoria Ostapenko $\cdot$ Natalia A. Popeko $\cdot$ Vladimir P. Skulachev $\cdot$ Maxim V. Skulachev

To view enhanced content go to www.advancesintherapy.com

Received: July 2, 2015 / Published online: December 11, 2015

(c) The Author(s) 2015. This article is published with open access at Springerlink.com

\section{ABSTRACT}

Introduction: This article presents the results of an international, multicenter, randomized, double-masked, placebo-controlled clinical study of Visomitin (Mitotech LLC, Moscow, Russian Federation) eye drops in patients with dry eye syndrome (DES). Visomitin is the first registered (in Russia) drug with a

V. V. Brzheskiy · E. L. Efimova · T. N. Vorontsova Saint-Petersburg State Pediatric Medical University, Saint Petersburg, Russian Federation

V. N. Alekseev

North-Western State Medical University Named After I.I. Mechnikov, Saint Petersburg,

Russian Federation

O. G. Gusarevich · K. N. Shaidurova

Novosibirsk State Medical University, Novosibirsk, Russian Federation

A. A. Ryabtseva - O. M. Andryukhina

Moscow Regional Research and Clinical Institute

(MONIKI), Moscow, Russian Federation

T. G. Kamenskikh · E. S. Sumarokova Saratov State Medical University Named After V.I. Razumovsky, Saratov, Russian Federation mitochondria-targeted antioxidant (SkQ1) as the active ingredient.

Methods: In this multicenter (10 sites) study of 240 subjects with DES, study drug (Visomitin or placebo) was self-administered three times daily (TID) for 6 weeks, followed by a 6-week follow-up period. Seven in-office study visits occurred every 2 weeks during both the treatment and follow-up periods. Efficacy measures included Schirmer's test, tear

\section{E. S. Miljudin}

Samara State Medical University, Samara, Russian Federation

\section{E. A. Egorov}

Pirogov Russian National Research Medical University, Moscow, Russian Federation

\section{O. I. Lebedev · A. V. Surov}

The Omsk State Medical Academy, Omsk, Russian Federation

\section{A. R. Korol - I. O. Nasinnyk}

The Filatov Institute of Eye Diseases and Tissue Therapy of the National Academy of Medical Sciences of Ukraine, Odessa, Ukraine

P. A. Bezditko · O. P. Muzhychuk Kharkiv National Medical University, Kharkiv, Ukraine 
break-up time, fluorescein staining, meniscus height, and visual acuity. Safety measures included adverse events, slit lamp biomicroscopy, tonometry, blood pressure, and heart rate. Tolerability was also evaluated.

Results: This clinical study showed the effectiveness of Visomitin eye drops in the treatment of signs and symptoms of DES compared with placebo. The study showed that a 6-week course of TID topical instillation of Visomitin significantly improved the functional state of the cornea; Visomitin increased tear film stability and reduced corneal damage. Significant reduction of dry eye symptoms (such as dryness, burning, grittiness, and blurred vision) was also observed. Conclusion: Based on the results of this study, Visomitin is effective and safe for use in eye patients with DES for protection from corneal damage.

Funding: Mitotech LLC.

Keywords: Dry eye syndrome;

Mitochondria-targeted antioxidants;

Ophthalmology; SkQ1

V. A. Vygodin · E. M. Karger $(\bowtie) \cdot$ O. N. Fedorkin . V. P. Skulachev

Belozersky Institute of Physico-Chemical Biology,

Lomonosov Moscow State University, Moscow,

Russian Federation

e-mail: karger@mitotech.ru

V. A. Vygodin · E. M. Karger · O. N. Fedorkin .

V. Ostapenko - N. A. Popeko - M. V. Skulachev Institute of Mitoengineering, Lomonosov Moscow

State University, Moscow, Russian Federation

E. V. Yani

Moscow Helmholtz Research Institute of Eye

Diseases, Moscow, Russian Federation

\section{A. Y. Savchenko}

Federal State Institution of Science Scientific Center of Biomedical Technology of the Federal Medical-Biological Agency of Russia, Svetlye Gory, Russian Federation

\section{INTRODUCTION}

Dry eye syndrome (DES) is a multifactorial disease of the tears and ocular surface that results in symptoms such as discomfort, visual disturbance, and tear film instability, with potential damage to the ocular surface. DES is accompanied by increased osmolarity of the tear film and inflammation of the ocular surface [1]. Increased osmolarity of the tear film can lead to morphological changes in the cornea and conjunctiva; activation of the inflammatory cascade can induce apoptotic cell death in the corneal epithelium and reduce the number of conjunctival goblet cells $[2,3]$. Prevalence of DES increases with age, significantly affecting the quality of life of senior patients $[1,4,5]$. Artificial tear treatment that is typically used to alleviate DES symptoms does not target the cause of the disease. Therefore, there is a need for drugs with reparative action that help to protect against the onset and progression of corneal lesions associated with DES $[5,6]$.

One promising therapeutic approach is protection of the anterior segment of the eye from oxidative stress, as oxidative stress and

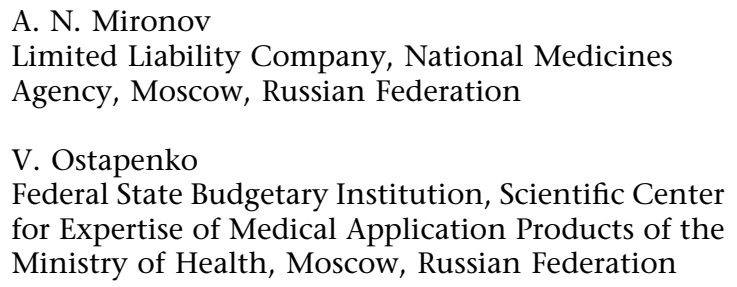

A. N. Mironov

Limited Liability Company, National Medicines Agency, Moscow, Russian Federation

V. Ostapenko

Federal State Budgetary Institution, Scientific Center for Expertise of Medical Application Products of the Ministry of Health, Moscow, Russian Federation

M. V. Skulachev

Biological Faculty, Lomonosov Moscow State University, Moscow, Russian Federation

M. V. Skulachev

Mitotech LLC, Moscow, Russian Federation 
reactive oxygen species (ROS) have been shown to play an important role in age-related eye diseases in general, and in DES in particular [7, 8]. One of the main sources of ROS in cells is mitochondria, which synthesize ATP and are the main consumers of molecular oxygen in the body $[9,10]$. Natural antioxidants are not capable of penetrating mitochondria and neutralizing the excessive amounts of free radicals generated by these organelles. Only recently a new class of chemical compounds-mitochondria-targeted antioxidants-capable of neutralizing mitochondrial ROS has been developed [11, 12]. The active ingredient SkQ1 in Visomitin (Mitotech LLC, Moscow, Russian Federation) ophthalmic solution belongs to this class of compounds and is capable of targeting and neutralizing mitochondrial $\operatorname{ROS}[13,14]$. Pre-clinical studies have shown that SkQ1 is a promising agent for treating ophthalmic diseases associated with oxidative stress [15-17]. Visomitin is the first drug containing SkQ1 that has been studied in clinical trials. In an initial clinical study, Visomitin demonstrated efficacy in treating signs and symptoms of DES compared to an artificial tear treatment used as a control [18]. Subsequently, Visomitin ophthalmic solution was approved in Russia in December 2011 and has since been marketed there as a prescription product. The purpose of the second clinical study that is the subject of this article was to assess the safety and tolerability of Visomitin and to evaluate the treatment and post-treatment efficacy of Visomitin compared with placebo (with the same artificial tears formulation used as a control in the initial Visomitin study) in patients suffering from DES with mild to moderate severity levels.

\section{METHODS}

A double-masked, randomized, placebo-controlled clinical trial of the efficacy and safety of Visomitin was conducted at 10 ophthalmic research centers in Russia and Ukraine. The study was conducted in accordance with good laboratory practice guidance, the Declaration of Helsinki, and Russian/Ukrainian regulatory requirements, and was registered with the Russian Ministry of Health. The study was approved by local ethics committees, and each subject was provided with a written informed consent to participate.

\section{Study Population}

The study included 240 patients (50 men and 190 women) aged 18-75 years demonstrating clinical signs of DES of mild to moderate severity levels, as determined by the investigator. Patients were excluded if they: had hypersensitivity reaction to the SkQ1 drug; had low visual acuity [VA; Sivtsev-Golovin scores [19] of $<0.3$ adjusted for worst seeing eye, with $1.0=$ good vision (equivalent to 20/20 on the Snellen chart) and approaching $0.0=$ poor vision (e.g., $0.1=20$ / 200 on the Snellen chart)]; had elevated intraocular pressure (IOP; >21 $\mathrm{mm} \mathrm{Hg}$ ); had acute inflammatory diseases of the eye; or had other diseases or conditions (e.g., diabetes, drug or alcohol addiction, pregnancy, breast feeding) that could have had an impact on treatment performance evaluation or safety.

There were no limitations for use of concomitant therapy during the study. Rare cases of ocular concomitant therapy usage are summarized in Table 1. Apart from the 11 patients mentioned in Table 1 , no other ocular concurrent eye drops or other medications were 
Table 1 Ocular concomitant medications

\begin{tabular}{|c|c|c|c|c|}
\hline \multirow{2}{*}{$\begin{array}{l}\text { Ocular concomitant } \\
\text { therapy/indication }\end{array}$} & \multicolumn{2}{|c|}{ Placebo $(n=120)$} & \multicolumn{2}{|c|}{ Visomitin $(n=120)$} \\
\hline & $\begin{array}{l}\text { Treatment } \\
\text { period }\end{array}$ & $\begin{array}{l}\text { Follow-up } \\
\text { period }\end{array}$ & $\begin{array}{l}\text { Treatment } \\
\text { period }\end{array}$ & $\begin{array}{l}\text { Follow-up } \\
\text { period }\end{array}$ \\
\hline Lubricant/dry eye & 0 & 6 & 0 & 2 \\
\hline Keratoprotector/dry eye & 1 & 1 & 0 & 1 \\
\hline Antioxidants/AMD & 0 & 0 & 1 & 1 \\
\hline
\end{tabular}

$A M D$ age-related macular degeneration

used. Other concomitant therapy included a broad range of medications prescribed for non-ocular concomitant diseases.

\section{Study Design}

All subjects who met the inclusion and exclusion criteria were randomized into two groups of 120 patients each. In the treatment group, patients were assigned Visomitin eye drops, and in the control group, patients were given placebo (in a double-masked manner). Patients were instructed to instill one drop per eye three times a day for 6 weeks. Visomitin eye drops contained $0.155 \mu \mathrm{g} / \mathrm{mL}(250 \mathrm{nM})$ SkQ1. The placebo consisted of benzalkonium chloride (0.1 mg), hypromellose (2 mg), sodium chloride $(9 \mathrm{mg})$, sodium dihydrogen phosphate dihydrate $(1.14 \mathrm{mg})$, and sodium dihydrogen phosphate dodecahydrate (0.96 mg) per $1 \mathrm{~mL}, \mathrm{pH} 6.5$, and was identical to the treatment drug Visomitin formulation except that the active ingredient SkQ1 was absent. During the treatment period, patients were required to visit their research center for examination every 2 weeks. After the end of the treatment period, patients discontinued instillations and visited the center every 2 weeks for another 6 weeks (follow-up observation period).
The study had 7 scheduled visits, with Visits 1-4 occurring during the 6 weeks of treatment, and Visits 5-7 occurring during the follow-up observation period (the next 6 weeks). At the visits, the following standard ophthalmological examinations were used to evaluate the effectiveness of the therapy: Schirmer's test, tear break-up time (TBUT) test, fluorescein staining, meniscus height, and VA. In addition, to evaluate safety, slit lamp biomicroscopy was conducted to assess pathological changes, tonometry was conducted to determine IOP, blood pressure and heart rate were measured, concomitant therapy drugs were noted, and complaints and adverse events (AEs) were recorded. After completion of treatment, patients and physicians were asked to evaluate the efficacy, tolerability, and residual efficacy (continued drug effect after treatment is terminated) using a visual analog scale (VAS) from 0 to 100 points, where $0=$ no effect and $100=$ very good effect.

Efficacy measurements for both eyes for each patient were pooled by group (treatment group or placebo group) to develop group statistics. For these parameters, positive response to treatment was assessed (i.e., number of eyes that showed an improvement by more than $5 \%$ from the initial level). 


\section{Study Procedures}

All criteria for evaluating the results of efficacy procedures were determined prospectively. The Schirmer's test was performed by applying certified test strips in the lower eyelid of each eye for $5 \mathrm{~min}$ without anesthesia. Schirmer's test results for tear production were evaluated as follows $[5,6]$ : wet segment of $\geq 25 \mathrm{~mm}=$ hyperlacrimation, $15-24 \mathrm{~mm}=$ normal, $10-14$ $\mathrm{mm}=$ mild level of tear production dysfunction, $\quad 5-9 \mathrm{~mm}=$ moderate,$\quad<5 \mathrm{~mm}$ = severe. The TBUT test for TBUT was performed in accordance with standard procedures. Results of the TBUT samples were evaluated as follows: $10 \mathrm{~s}$ or more $=$ normal [20], 5-9 s = mild level of TBUT dysfunction, 3-4 s = moderate, $<3 \mathrm{~s}=$ severe $[5,6]$. Corneal staining was performed using fluorescein strips, with the cornea divided into five zones: central, superior, inferior, nasal, and temporal. The number and size of corneal lesions were evaluated separately for each zone using the following scale: no staining $=0$ points, $1-15$ punctate staining areas $=1$ point, $>15$ areas of punctate staining or one continuous area $=2$ points, $>15$ areas of punctate staining and one or more continuous area $=3$ points. The total damage to the cornea was determined by adding the scores for all five zones. Tear meniscus height was determined before other procedures were performed. VA was determined using Sivtsev-Golovin tables illuminated at 600-1000 lux at a $5 \mathrm{~m}$ distance.

\section{Statistical Analysis}

Two-sided levels of significance and confidence intervals were calculated and reported, and a statistical level of significance of 0.05 was used (unless otherwise indicated). For efficacy parameters, significance assessments were performed using a modified $t$ test (with Fischer arcsin-frequency conversion for frequency variables), $\chi^{2}$ test with Mantel-Haenszel correction, and Fisher's exact test. Statistical analysis was performed using SAS software version 6.12 (SAS Institute Inc., Cary, NC, USA). No changes were made to the methodology after the trial began. One post-study subgroup analysis was conducted for baseline total corneal damage (elevated or not elevated), as discussed in this article. No other subgroup analyses were conducted, and no interim or other adjusted analyses were conducted.

\section{RESULTS}

\section{Subject Demographics and Disposition}

The Visomitin treatment group and the placebo group were statistically comparable in terms of gender and age: both groups were dominated by female patients, and average age in the placebo group was 46 years and in the treatment group was 47 years (Table 2). All randomized subjects received treatment. In addition to the 235 patients who completed the study (116 in the placebo group and 119 in the Visomitin treatment group), 3 patients assigned to the placebo group withdrew consent to participate in the study for personal reasons, 1 patient assigned to the placebo group dropped out due to an $\mathrm{AE}$ of allergic conjunctivitis, and 1 patient assigned to the Visomitin group dropped out as a result of a serious AE (SAE; see "Safety Results").

\section{Safety Results}

AEs were typical of those reported by patients with DES and included burning sensation after 
Table 2 Demographic and tear function parameters at baseline

\begin{tabular}{llll}
\hline Characteristic & \multicolumn{2}{l}{ Group } & \\
\cline { 2 - 4 } & Visomitin $(\boldsymbol{n}=\mathbf{1 2 0})$ & Placebo $(\boldsymbol{n}=\mathbf{1 2 0})$ & All subjects $(\boldsymbol{n}=\mathbf{2 4 0})$ \\
\hline Age, years, mean \pm SD & $47.5 \pm 1.4$ & $46.3 \pm 1.4$ & $46.9 \pm 0.97$ \\
Sex, female, $n(\%)^{\mathrm{a}}$ & $95(79.1 \%)$ & $95(79.1 \%)$ & $190(79.1 \%)$ \\
Baseline tear function $(\mathrm{ITT})$, mean \pm SD & & \\
Schirmer's test $^{\mathrm{b}}$ & $11.7 \pm 0.6$ & $11.1 \pm 0.5$ & $11.6 \pm 0.4$ \\
TBUT $^{c}$ & $6.8 \pm 0.2$ & $7.2 \pm 0.2$ & $7.0 \pm 0.2$ \\
\hline
\end{tabular}

ITT Intent-to-treat population, SD Standard deviation, TBUT Tear break-up time

a Number of subjects, values in parentheses equal the percent of the number of subjects in the treatment group

b Schirmer's test was measured in $\mathrm{mm}$ and performed in accordance with standard procedures. Mean at baseline is the average of all eyes

c TBUT was measured in seconds and performed in accordance with standard procedures. Mean at baseline is the average of all eyes

instillation of the test drug, which is commonly reported after use of artificial tear formulations in the conjunctival cavity $[5,6]$. One SAE, a fatal myocardial infarction that was considered unrelated to drug treatment, was reported in the Visomitin group during the follow-up observational period. In addition, 55 AEs were reported: 21 AEs in the placebo group and 34 AEs in the Visomitin group. AEs considered definitely related to the study drug, in addition to burning after instillation (which occurred in similar numbers in both treatment groups), included eye redness after instillation and scratchiness in the Visomitin group, and blurring vision after instillation, and itchiness, redness, and hypersensitivity reaction including papular eruption on the hands, neck, and chest in the placebo group. Other AEs that were considered probably or possibly related to drug treatment included lacrimation or tingling on instillation, eye dryness, and itchiness in the Visomitin group, and prominent eyelid and conjunctiva hyperemia, swelling similar to allergy reaction, and film-like coating, tingling, lacrimation, or scratchiness after instillation in the placebo group.
Table 3 Adverse events

\begin{tabular}{llc}
\hline Classification of AEs by & \multicolumn{2}{l}{ Reported AEs in group } \\
\cline { 2 - 3 } possible association with drug & $\begin{array}{l}\text { Placebo } \\
(\boldsymbol{n}=\mathbf{1 2 0})\end{array}$ & $\begin{array}{l}\text { Visomitin } \\
(\boldsymbol{n}=\mathbf{1 2 0})\end{array}$ \\
\hline Not associated & 3 & 8 \\
Association uncertain & 2 & 8 \\
Possibly associated & 9 & 8 \\
Probably associated & 3 & 4 \\
Associated & 4 & 6 \\
Total & 21 & 34 \\
\hline
\end{tabular}

$A E s$ adverse events

AEs classified by the degree of relationship to the drug are shown in Table 3. One AE of allergic reaction that led to discontinuation of treatment was reported (in the placebo group). No other AEs resulted in discontinuation of treatment. There were no AEs of elevated IOP, blood pressure, or heart rate. Tolerability test results using a tolerability VAS showed that Visomitin was well tolerated: the average tolerability rating on a scale from 0 to $100 \%$ (with $0=$ absolutely intolerable and $100=$ very well tolerated) was approximately 90\%. Based on the safety results, Visomitin was categorized 
as safe for use in patients with DES and well tolerated.

\section{Visual Acuity}

Patients had relatively high VA levels at the time of enrollment prior to treatment (average uncorrected Sivtsev-Golovin VA score was $0.63 \pm 0.02$ in the placebo group and $0.62 \pm 0.02$ in the Visomitin group; average corrected VA was close to 1 in both groups). VA statistically significantly $(P<0.05)$ improved over the 6 weeks of therapy in both groups. At Visit 4, the average uncorrected VA was $0.64 \pm 0.02$ in the placebo group and $0.63 \pm 0.02$ in the Visomitin group, with no statistically significant difference between the groups. This VA improvement during the treatment period was probably due to the effect of hypromellose present in both the Visomitin and placebo formulations.

\section{Meniscus Height}

Meniscus height at the time of enrollment was $256.3 \pm 9.3 \mu \mathrm{m}$ in the placebo group and $259.8 \pm 8.1 \mu \mathrm{m}$ in the Visomitin group. At Visit 4 , meniscus height statistically significantly $(P<0.001)$ improved in both groups $(308.3 \pm 9.8 \mu \mathrm{m}$ in the placebo group and $298.1 \pm 8.4 \mu \mathrm{m}$ in the Visomitin group). At Visit 7 , meniscus height scores worsened in both groups $(262.6 \pm 7.8 \mu \mathrm{m}$ in the placebo group and $274.0 \pm 6.9 \mu \mathrm{m}$ in the Visomitin group), with no statistically significant difference between the groups.

\section{Schirmer's Test}

Schirmer's test scores at baseline averaged $11.1 \pm 0.5 \mathrm{~mm}$ in the placebo group and $11.7 \pm 0.6 \mathrm{~mm}$ in the Visomitin group, which corresponds to a mild level of tear production dysfunction. At baseline, several patients suffered from hyperlacrimation, most patients showed a mild level of tear production dysfunction, a third of the population suffered from a moderate level of tear dysfunction, and about $10 \%$ were assigned to the 'severe' category according to the Schirmer's test. By the end of the treatment (Visit 4), the average Schirmer's test scores in both groups improved (increased) significantly $(P<0.001) \quad$ to $13.7 \pm 0.4 \mathrm{~mm}$ in the placebo group and $14.2 \pm 0.5 \mathrm{~mm}$ in the Visomitin group. At the end of the observation period (Visit 7), scores decreased almost to baseline levels, averaging $11.6 \pm 0.4 \mathrm{~mm}$ in the placebo group and $12.2 \pm 0.5 \mathrm{~mm}$ in the Visomitin group, with the difference between the groups not significant. These data suggest that the effect of Visomitin on tear production was positive, but did not dominate the effect of hypromellose over the 6-week treatment period.

\section{TBUT Test}

The TBUT test is an important metric for DES severity as it reflects the overall tear quality throughout all layers of the tear film. At baseline, patients in both the Visomitin and placebo groups were predominantly assigned to the mild category of tear production dysfunction. TBUT test averages were $7.2 \pm 0.2 \mathrm{~s}$ in the placebo group and $6.8 \pm 0.2 \mathrm{~s}$ in the Visomitin group. Change in TBUT test results from baseline throughout the study visits is shown in Fig. 1. By the end of the treatment period (Visit 4), TBUT in patients treated with Visomitin increased (improved) slightly more than in patients in the placebo group (change from baseline was $2.9 \pm 0.3 \mathrm{~s}$ in the placebo group and $3.1 \pm 0.3 \mathrm{~s}$ in the Visomitin group, although these differences 


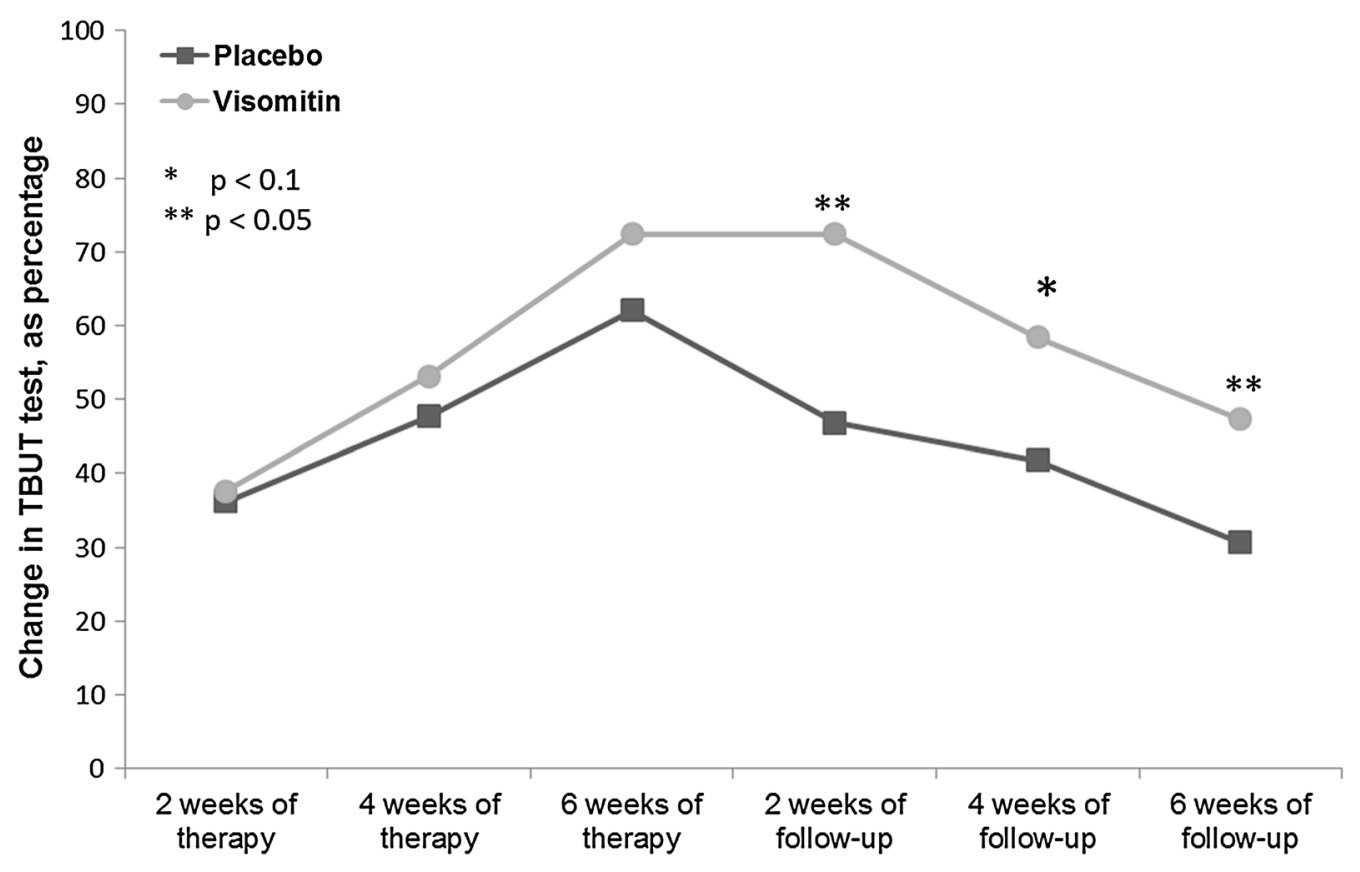

Fig. 1 TBUT test change from baseline as percentage: all patients. TBUT scores improved (increased) in both the Visomitin and placebo groups; greater improvement was seen in the Visomitin group. Statistically significant differences were observed between the two groups at

were not statistically significant). This effect persisted for at least 2 weeks after drug instillations were stopped. Some level of improvement of TBUT was maintained in patients in the Visomitin group even at the end of the follow-up observation period (Visit 7).

\section{Evaluation of Corneal Damage}

In this study, the fluorescein staining technique was used to evaluate the area of damage in five different zones of the cornea (central, superior, inferior, nasal, and temporal) and in the cornea overall (total damage score, as a sum of zone scores). The maximum possible total damage score was 15, and a total score of 3 or higher was considered abnormal. The damage level in the central zone of the cornea is one of the most interesting indicators in DES patients, as this
Weeks 2 and $6(P<0.05)$ during the follow-up observation period. Differences between the groups at Week 4 during follow-up $(P<0.1)$ are also shown $(P$ values compare results between the two groups). TBUT tear break-up time

zone is most prone to the influence of environmental factors. Figure 2 shows the average staining scores for the central zone of the cornea, and Fig. 3 shows the average total staining scores. Both central corneal zone damage and total corneal damage were significantly $(P<0.001)$ reduced during the treatment period in both the Visomitin and placebo groups. During the follow-up observation period, a significant post-treatment effect was seen for Visomitin compared to placebo. The difference between the groups for central corneal fluorescein staining was statistically significant 2 weeks after treatment was stopped, at Visit 5 $(P<0.01)$ and at Visit $6 \quad$ (4 weeks post-treatment, $\quad P<0.05$; Fig. 2). For total corneal fluorescein staining, the difference between the groups was statistically significant at Visits 5 and $6(P<0.01$ at both time points; 


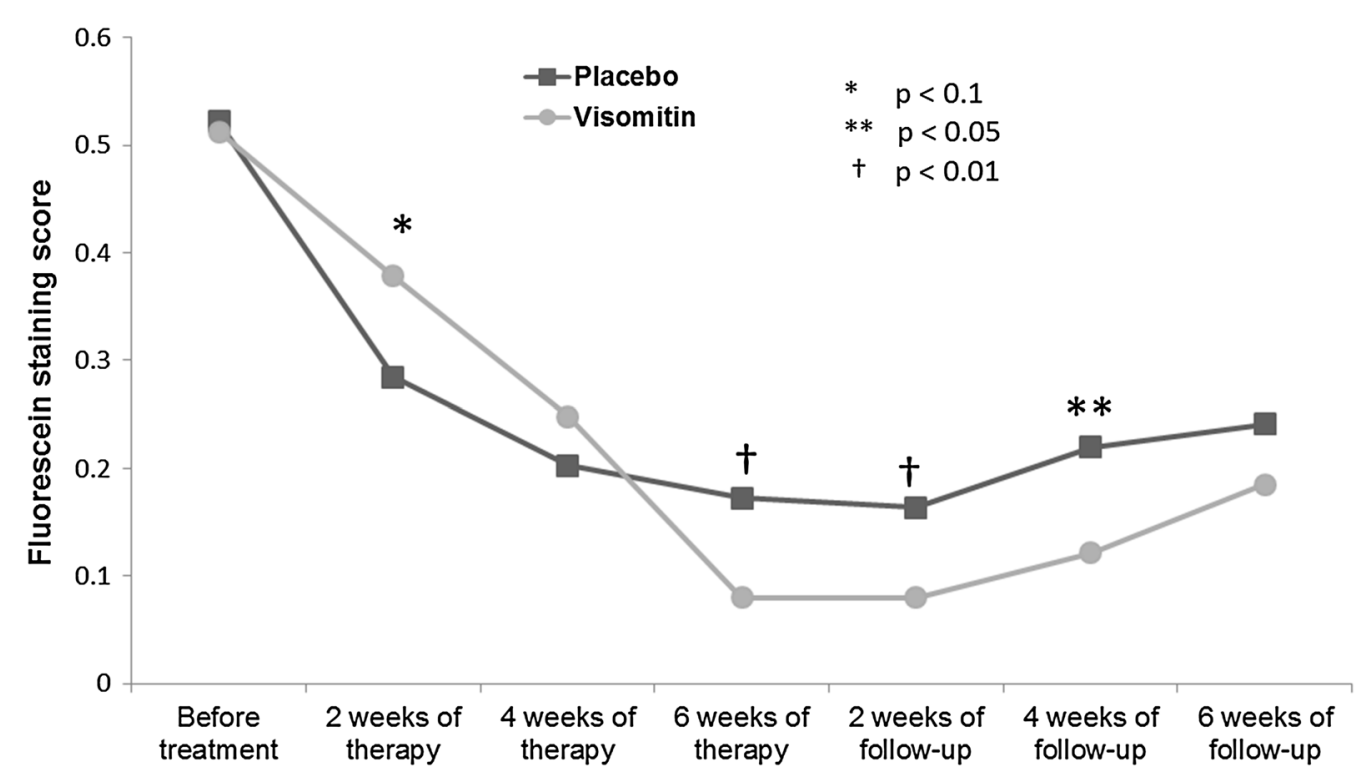

Fig. 2 Central corneal fluorescein staining: all patients. Central corneal staining improved (decreased) more in the Visomitin group than in the placebo group, with statistically significant differences between the two groups at Week 6

Fig. 3). Similar results were obtained for the other corneal zones (data not shown).

\section{Efficacy in Patients with Elevated Corneal Damage}

For an additional post-study analysis, all patient eyes were assigned to two subgroups according to the degree of their baseline total corneal damage: mild damage (below 3 points), and elevated damage (3 points or more). Sixty-five eyes treated with placebo and 57 eyes treated with Visomitin were assigned to the elevated corneal damage subgroup, which demonstrated the most pronounced effect of Visomitin.

Average baseline Schirmer's test scores for the 'elevated corneal damage' subgroup were $10.1 \pm 1.0 \mathrm{~mm}$ for patients treated with placebo and $12.4 \pm 1.2 \mathrm{~mm}$ for patients treated with Visomitin. At the end of the treatment period, Schirmer's test score averages increased during the treatment period and at Weeks 2 and 4 of the follow-up observation period ( $P$ values compare results between the two groups)

(improved) to $13.2 \pm 0.9 \mathrm{~mm}$ in the placebo group and $14.6 \pm 0.6 \mathrm{~mm}$ in the Visomitin group. After another 6 weeks of the follow-up observation period, Schirmer's test scores returned to approximately baseline values in both groups. Differences between the placebo and Visomitin groups were not significant at any of the visits for the elevated corneal damage subgroup. However, positive response to treatment as reflected by an increase in Schirmer's test scores by the end of the treatment period was $72 \%$ for placebo patients and $86 \%$ for Visomitin patients $(P<0.1)$.

Figure 4 shows the change from baseline in TBUT test results in patients with elevated corneal damage as a percentage. In this subgroup, for patients treated with Visomitin, average TBUT test results increased (improved) by $5 \mathrm{~s}$ by the end of treatment, compared to $4 \mathrm{~s}$ for patients receiving placebo. By the end of the follow-up observation period, the TBUT test 


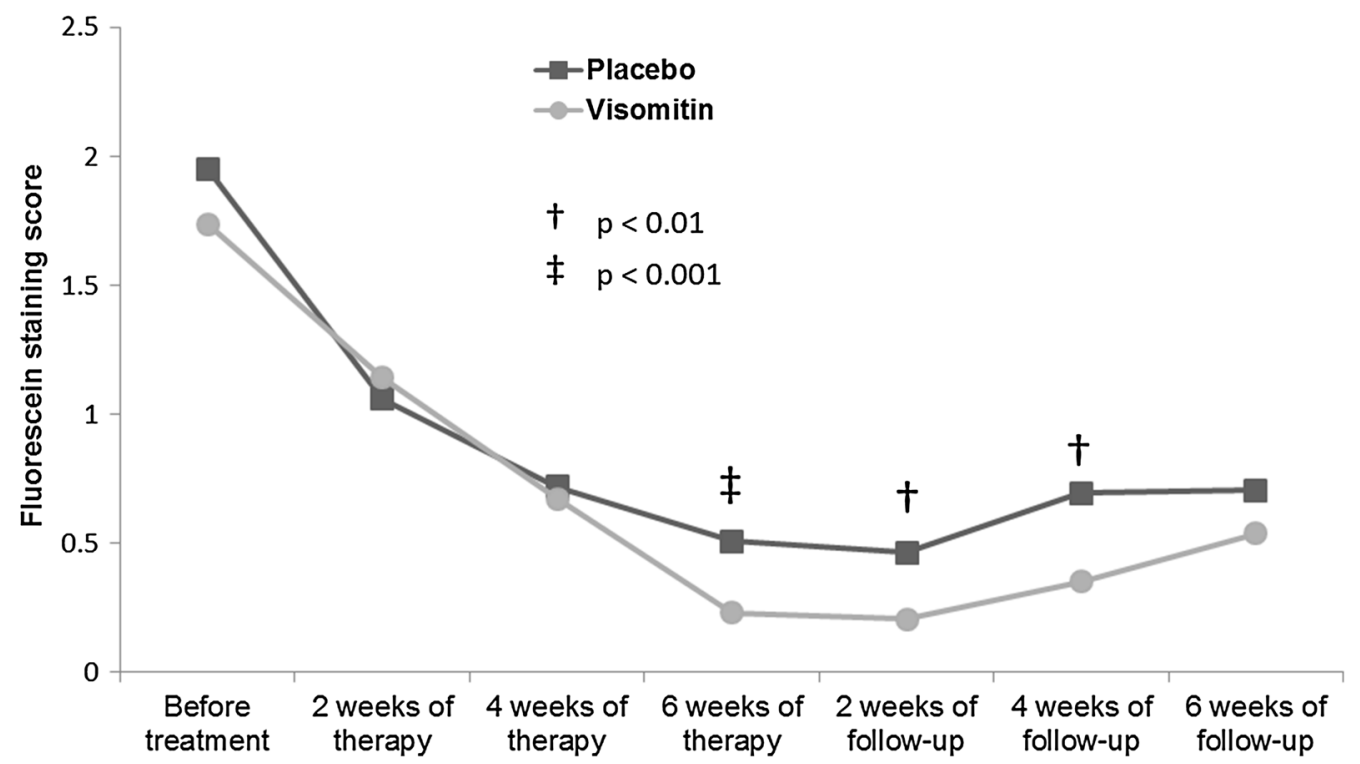

Fig. 3 Total corneal fluorescein staining: all patients. Total corneal staining improved (decreased) more in the Visomitin group than in the placebo group, with statistically significant differences between the two groups at Week 6

score average for the Visomitin patients in this subgroup was $3 \mathrm{~s}$ higher than it was prior to treatment, compared to $1.5 \mathrm{~s}$ for patients receiving placebo.

Corneal fluorescein staining results were notable for the subgroup of patients with elevated corneal damage. Figure 5 shows average central corneal staining scores in the elevated corneal damage subgroup, and Fig. 6 shows average total corneal staining in this subgroup. Corneal staining (corneal damage) was significantly reduced in patients treated with Visomitin compared to patients receiving placebo after 6 weeks of treatment, and this effect remained for the next 6 weeks of follow-up observation. Response to treatment (positive response $=$ number of eyes with $>5 \%$ improvement from baseline) for central corneal staining at the end of treatment (Visit 4) was $54 \%$ in placebo patients and $72 \%$ in Visomitin patients $(P<0.05)$. Similar trends were observed during the treatment period and at Weeks 2 and 4 of the follow-up observation period ( $P$ values compare results between the two groups)

in other areas of the cornea. Thus, corneal staining results for Visomitin showed significant therapeutic effect in protecting the cornea from damage in the general population of DES patients when compared to patients who received placebo, and this therapeutic effect was especially pronounced in the elevated corneal damage subgroup.

\section{Evaluation of Symptom Scores}

All symptoms were evaluated using a scale reflecting the frequency of symptoms: the greater the frequency of the symptom, the higher the score. Symptom changes (relative to the baseline visit) for dryness, grittiness, blurred vision, and worst symptom (estimated as the symptom with the highest score at baseline) are shown in Fig. 7 (for all patients) and Fig. 8 (for the elevated corneal damage subgroup). Statistical analysis indicated that 


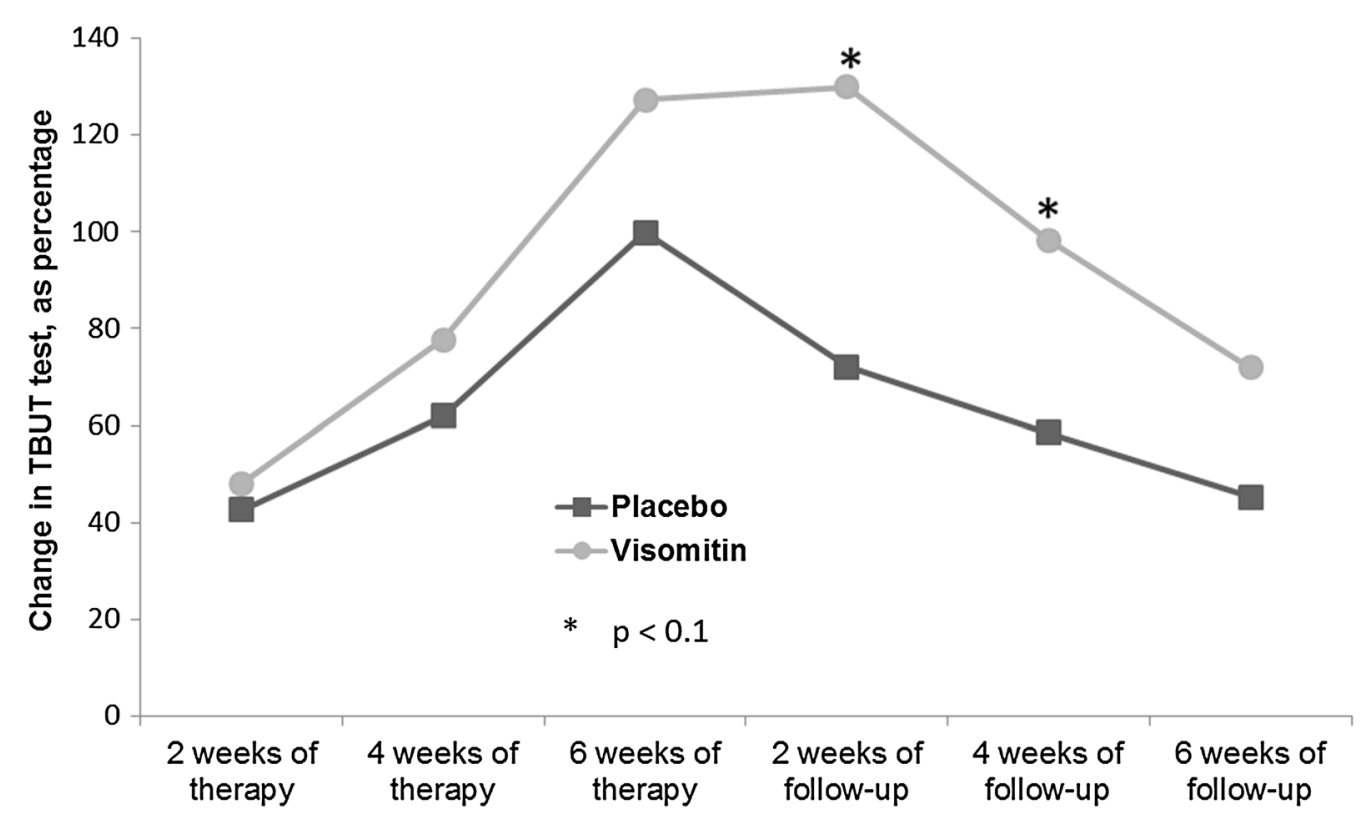

Fig. 4 TBUT test, change from baseline as percentage in patients with elevated corneal damage. TBUT scores improved (increased) in this subgroup of patients with elevated corneal damage both in the Visomitin and placebo groups, with statistically significant differences between the two groups at Weeks 2, 3, and 4 of the follow-up

blurred vision, dryness, grittiness, burning, and eye fatigue decreased during treatment in both the Visomitin and placebo groups, but the decrease in the Visomitin group was more pronounced, especially during the follow-up observation period. The differences between the groups were statistically significant for blurred vision 4 weeks after treatment was stopped (Visit $6, P<0.05$ ) and 6 weeks post-treatment (Visit $7, P<0.01)$; for dryness $(P<0.1)$ at Visits 6 and 7 (6 weeks post-treatment); for grittiness $(P<0.1)$ at Visit 7 ; for worst symptom $(P<0.01)$ at Visit 5 , and at Visits 6 and $7(P<0.001)$. This decrease was even greater for the elevated corneal damage subgroup (Fig. 8), with statistically significant differences between the groups for dryness 4 weeks after treatment was stopped (Visit 6, $P<0.1$ ); for grittiness after 6 weeks of therapy (Visit $4, P<0.05$ ) and 2 and observation period; greater improvement, for a longer time period, was seen in the Visomitin group, and was greater in this subgroup than for all patients combined ( $P$ values compare results between the two groups). TBUT Tear break-up time

4 weeks post-treatment (Visits 5 and $6, P<0.01$ and $P<0.05$, respectively); and for blurred vision during treatment at Visit $2(P<0.01)$, Visit $3(P<0.05)$, and Visit $4(P<0.01)$, and post-treatment at Visits 5, 6, and $7(P<0.001)$. Worst symptom score also showed superior efficacy of Visomitin compared to the placebo group during the follow-up observation period, with differences between the groups during treatment at Visit $3 \quad(P<0.1)$ and Visit 4 $(P<0.05)$ and post-treatment at Visits 5,6 , and $7(P<0.01)$.

\section{DISCUSSION}

The results of recent studies indicate that clinical and biological signs of DES often do not match the severity of symptoms [1, 22, 23]. Despite the large number of diagnostic 


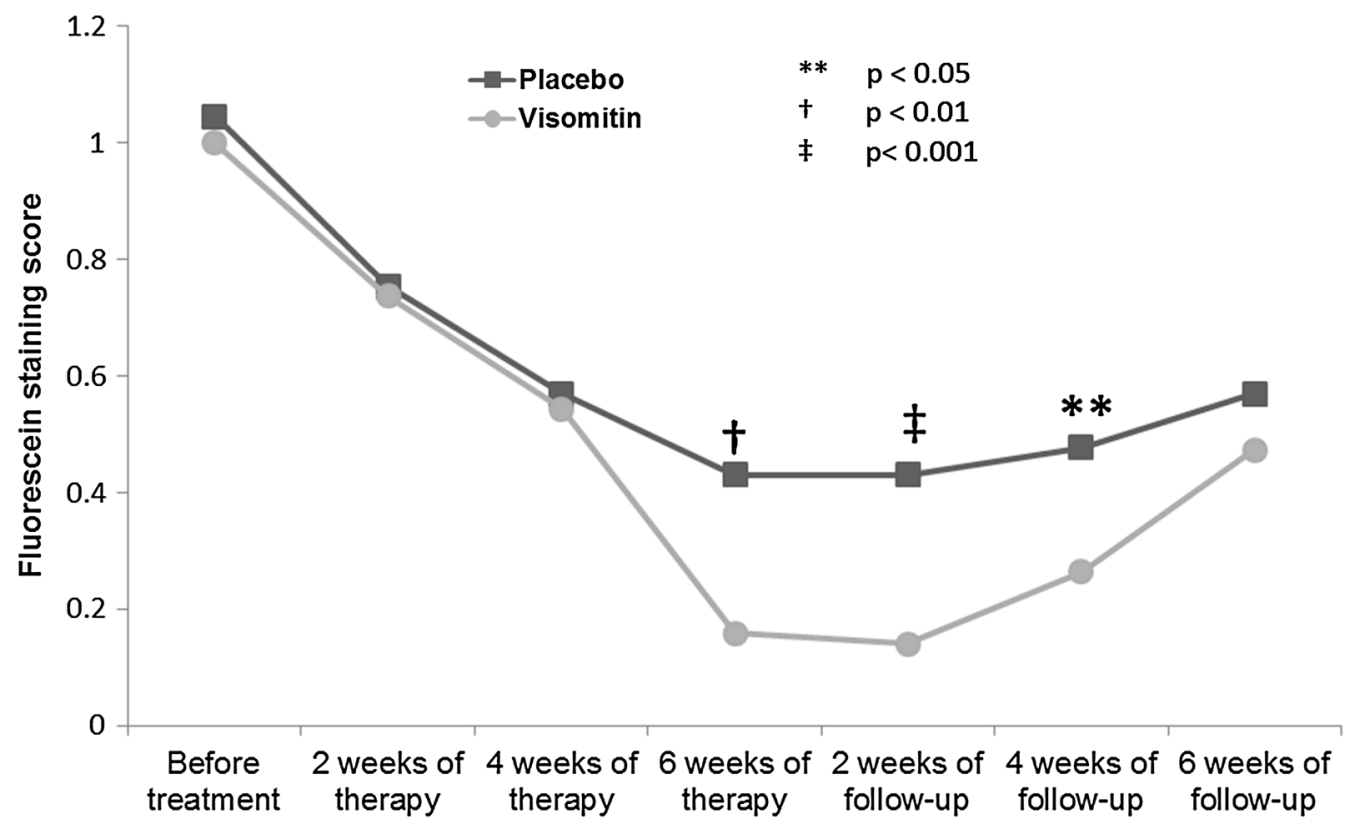

Fig. 5 Central corneal fluorescein staining in patients with elevated corneal damage. Central corneal staining improved (decreased) more in the Visomitin group than in the placebo group in this subgroup of patients with elevated corneal damage, with statistically significant differences

techniques, there is no single 'gold standard' that can determine the severity of the syndrome and evaluate therapy effectiveness. For example, the commonly used Schirmer's test is not sufficient to uniformly assess severity of DES. Basal tear deficit can be compensated by reflex tear production, in which case the Schirmer's test result will not indicate decline and sometimes will even show an increase.

Another standard method for diagnosing DES-corneal fluorescein staining-also has its drawbacks. Elevated levels of staining sometimes occur in patients who do not have DES [24]. Moreover, reproducibility of corneal and conjunctival staining has been shown to be rather low [25]. A study that involved 300 patients found tear osmolarity to be the best marker of DES severity [26]. However, conjunctival and corneal stainings were shown to have the highest correlation with DES between the Visomitin and placebo groups at Week 6 during the treatment period and at Weeks 2 and 4 of the follow-up observation period ( $P$ values compare results between the two groups)

severity among commonly used diagnosis techniques. According to ophthalmologists of the ODISSEY European Consensus Group, which studied 14 commonly used DES diagnosis techniques, a joint analysis of symptom scores and fluorescein staining scores is usually sufficient for assessing DES severity [21].

Taking the above DES assessment methods into consideration, a variety of measurement techniques was used in the randomized, double-masked, placebo-controlled clinical trial of Visomitin. Symptoms scores, slit lamp exam data, Schirmer's test data, TBUT, and corneal fluorescein staining data were assessed in patients with mild to moderate DES levels during the course of this study.

The study results of corneal fluorescein staining (Figs. 2 and 3) are of particular interest. It was demonstrated that the 


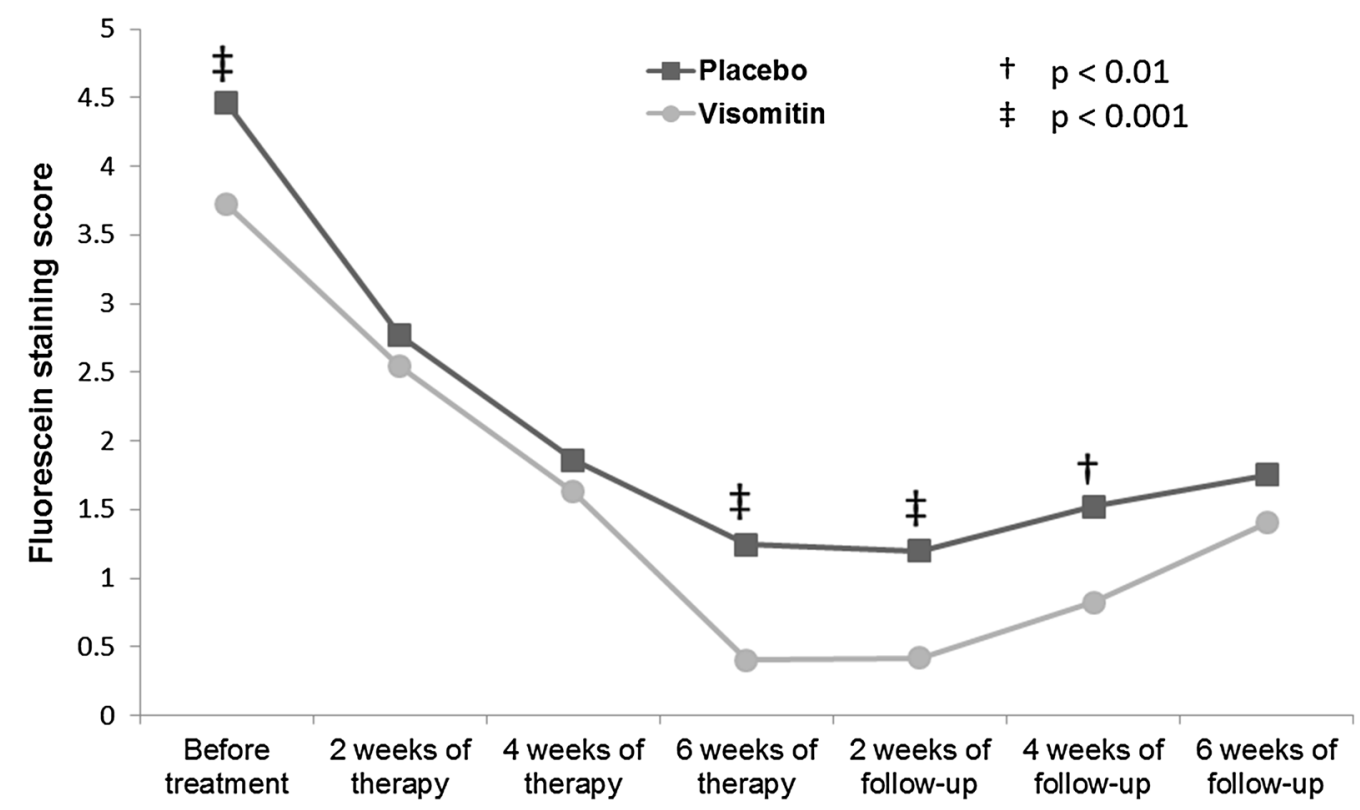

Fig. 6 Total corneal fluorescein staining in patients with elevated corneal damage. Total corneal staining improved (decreased) more in the Visomitin group than in the placebo group in this subgroup of patients with elevated corneal damage, with statistically significant differences

Visomitin group had significantly less corneal damage in the central zone after the sixth week of treatment than the placebo group. Furthermore, this effect persisted for at least 4 weeks after the therapy was terminated.

This statistically significant difference between the efficacy of Visomitin and placebo was mainly driven by the subgroup of patients with more elevated corneal damage (with total fluorescein staining scores $\geq 3$ at baseline). Based on Sullivan et al. [26], these patients can be described as a subgroup with a moderate degree of dry eye. Interestingly, TBUT values in this subgroup were significantly higher (improved) in patients treated with Visomitin than in patients treated with placebo at the end of treatment and for the duration of the follow-up observation period (Fig. 4). Results also showed improvement in the symptom of blurred vision in this subgroup (Fig. 7b). Tutt between the Visomitin and placebo groups at Week 6 during the treatment period and at Weeks 2 and 4 of the follow-up observation period ( $P$ values compare results between the two groups)

et al. [27] argue that blurred vision, typical for patients with dry eye, is associated with a decrease of tear film stability and, therefore, should correlate with TBUT measurements.

The results of this study showed that SkQ1 eye drops did not affect tear production levels: Schirmer's test scores increased in both the Visomitin and placebo groups and did not differ significantly between the groups. According to Lemp [28], results of the Schirmer's test may significantly vary even throughout a single visit. Also, the Schirmer's test appears to be more reproducible in patients with low test values, that is, more severe disease. Therefore, it is believed that the applicability of the Schirmer's test in patients with mild to moderate dry eye is limited.

It is known that inflammation plays an important role in the pathogenesis of DES [28]. An increase in soluble mediators 

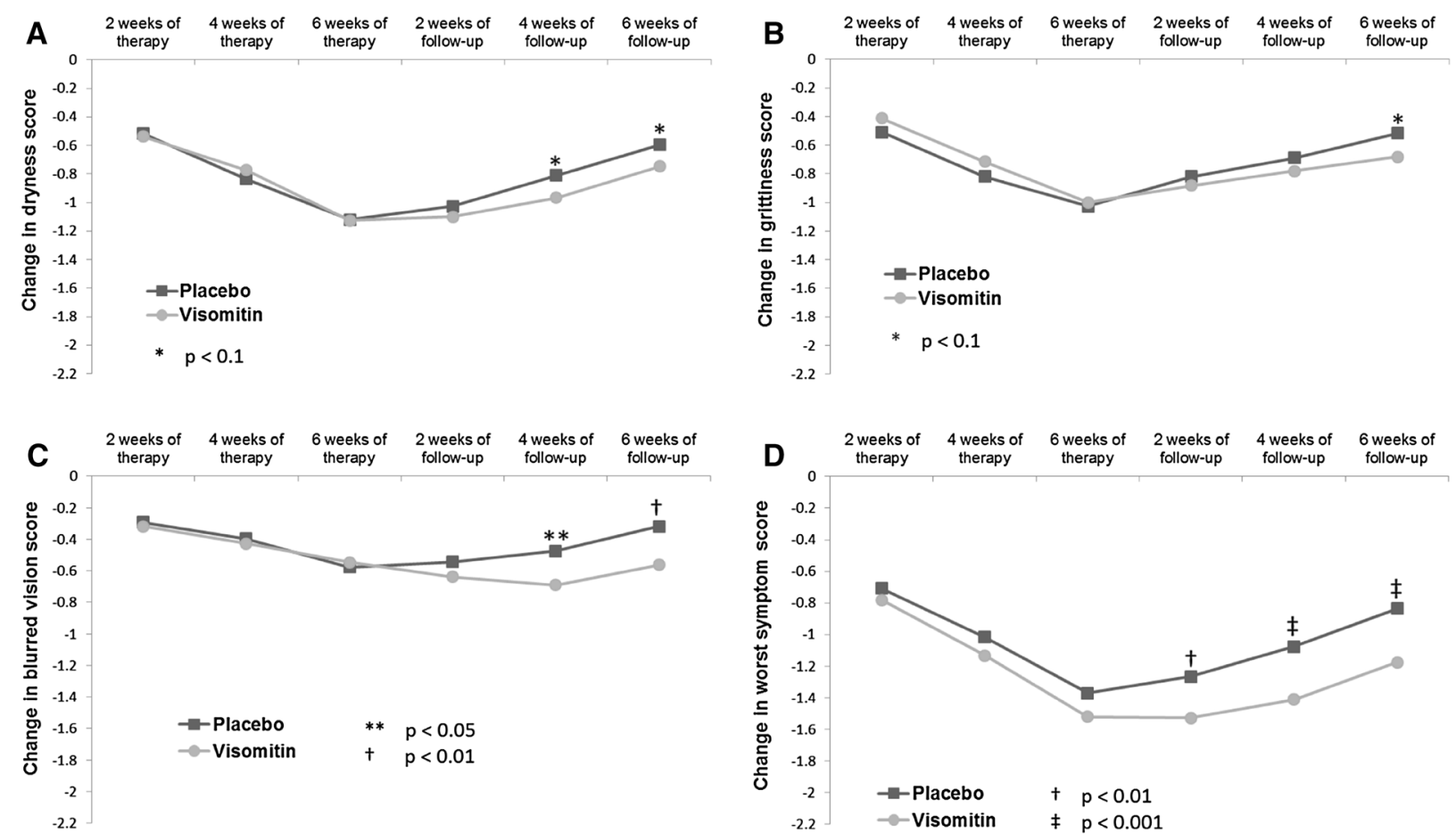

Fig. 7 Symptom score change from baseline-all patients: a dryness, b grittiness, c blurred vision, and d worst symptom. For all four symptoms (dryness, grittiness, blurred vision, and worst symptom), scores decreased (improved) in both the Visomitin and placebo groups through Week 6 of the treatment period, then increased during the 6 weeks of the follow-up observational period. There was no statistically significant difference between the

(cytokines and proteases) in the tear fluid, adhesion molecule expression by the conjunctival epithelium, and $\mathrm{T}$ cell infiltration of the conjunctiva have all been observed in dry eye patients [29]. It was previously shown that the anti-inflammatory effect of SkQ1, which inhibits the NF-kB signaling pathway, can lead to a decrease in the level of expression of adhesion molecules and in leukocyte infiltration [30]. It is probable that the anti-inflammatory effect of SkQ1 plays an important role in reducing corneal damage in patients with DES.

These data indicate that Visomitin not only has a moistening effect due to the presence of

two groups during the treatment period, and scores were better (lower) for the Visomitin group during the 6-week follow-up observational period. Statistically significant differences occurred in favor of the Visomitin group for blurred vision (Weeks 4 and 6 of the follow-up period) and for worst symptom (Weeks 2, 4, and 6 of the follow-up period; $P$ values compare results between the two groups)

hypromellose, but also that the active compound SkQ1 restores corneal cell function and tear film stability, thus directly addressing the causes of DES. Improvements in both symptoms and corneal staining associated with DES are attributable to Visomitin treatment, suggesting a beneficial therapeutic effect of the drug. Furthermore, post-therapy efficacy of Visomitin for TBUT, corneal staining, and symptoms over 4 weeks of the follow-up observation period, especially in patients with moderate dry eye, shows a clear advantage of Visomitin over artificial tear treatments.

One possible limitation of the study was that the severity of dry eye was determined by the 

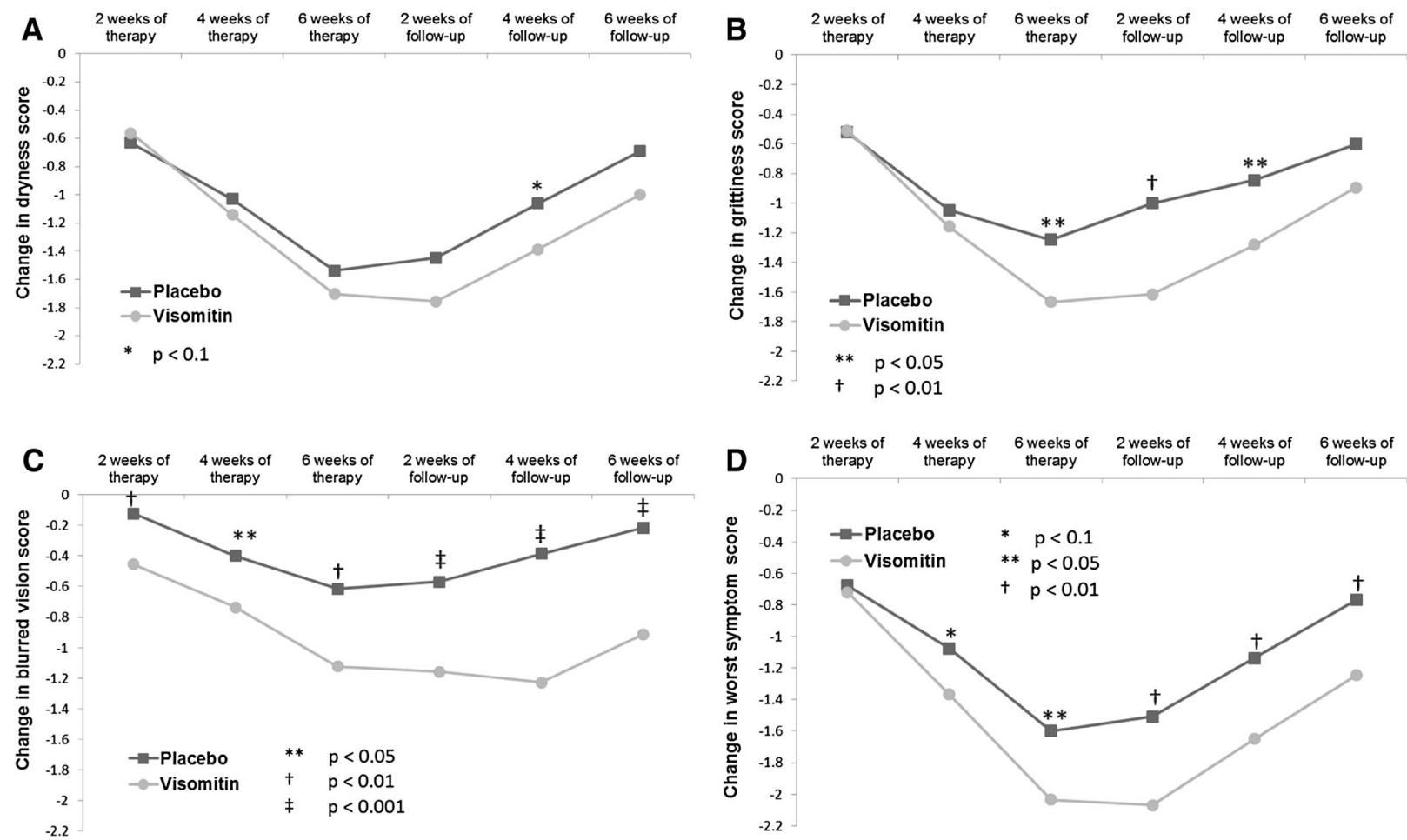

Fig. 8 Symptom score change from baseline in patients with elevated corneal damage: a dryness, b grittiness, c blurred vision, and $\mathbf{d}$ worst symptom. For all four symptoms (dryness, grittiness, blurred vision, and worst symptom), scores decreased (improved) in the subgroup of patients with elevated corneal damage in both the Visomitin and placebo groups through Week 6 of the treatment

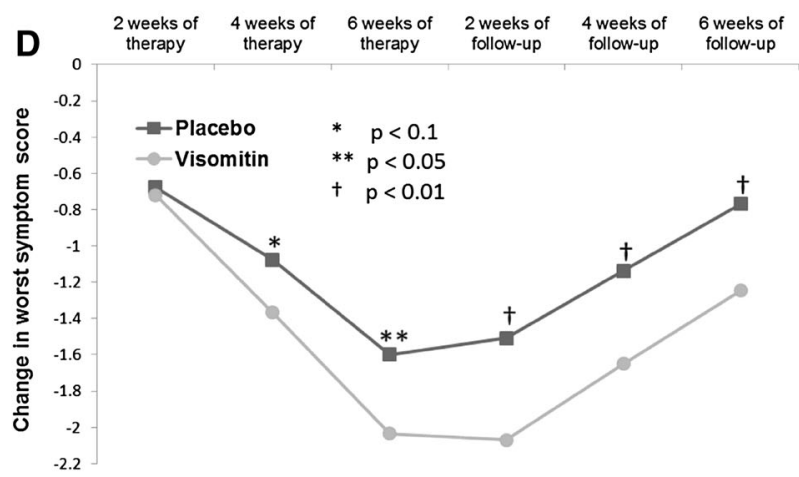

period, then increased during the 6 weeks of the follow-up observational period. Scores were generally better (lower) for the Visomitin group, with statistically significant differences in favor of the Visomitin group at most time points, especially for grittiness, blurred vision and worst symptom ( $P$ values compare results between the two groups)

investigator and not quantified or specified earlier in the study as to how the investigators would decide the subject's dry eye was mild or moderate in severity. However, the better results in the higher corneal staining subgroup suggest that the population was mixed; also, no standardized definition or selection criteria of DES severity currently exists. Also, there are limitations of some of the dry eye tests themselves (e.g., Schirmer's test, corneal fluorescein staining), as mentioned above; using several dry eye tests compensates somewhat for the test limitations. In addition, seasonality may contribute to a reduction in the effects of dry eye treatments as the drier season

progresses. Also, environmental factors, such as subjects' increased use of computers or reading or exposure to tobacco smoke or wind, may also decrease the effects of drug treatments.

\section{CONCLUSIONS}

Visomitin has several important benefits for dry eye patients. Visomitin has been shown to be safe and well tolerated in patients with DES. Visomitin exhibited a more pronounced and stable post-therapy effect in patients with DES than placebo. Visomitin is highly effective in protecting patients with DES from corneal damage. 


\section{ACKNOWLEDGMENTS}

This study was funded by Mitotech LLC. The article processing charges and open access fee for this publication were funded by Mitotech S.A. Editorial services were provided by Linda Stein, Ora, Inc. Editorial services were funded by Mitotech S.A. All named authors meet the International Committee of Medical Journal Editors (ICMJE) criteria for authorship for this manuscript, take responsibility for the integrity of the work as a whole, and have given final approval for the version to be published.

Disclosures. All authors participated in conducting this study and received compensation for their work. M. Skulachev is the general director of Mitotech LLC, the company that funded this study and markets Visomitin in Russia. V. Brzheskiy, E. Efimova, T. Vorontsova, V. Alekseev, O. Gusarevich, K. Shaidurova, A. Ryabtseva, O. Andryukhina, T. Kamenskikh, E. Sumarokova, E. Miljudin, E. Egorov, O. Lebedev, A. Surov, A. Korol, I. Nasinnyk, P. Bezditko, O. Muzhychuk, V. Vygodin, E. Yani, A. Savchenko, E. Karger, O. Fedorkin, A. Mironov, V. Ostapenko, N. Popeko, and V. Skulachev have nothing further to disclose.

Compliance with ethics guidelines. The study was conducted in accordance with good laboratory practice guidance, the Declaration of Helsinki, and Russian/Ukrainian regulatory requirements, and was registered with the Russian Ministry of Health. The study was approved by local ethics committees, and each subject was provided with a written informed consent to participate.

Open Access. This article is distributed under the terms of the Creative Commons
Attribution-NonCommercial 4.0 International License (http://creativecommons.org/licenses/ by-nc/4.0/), which permits any noncommercial use, distribution, and reproduction in any medium, provided you give appropriate credit to the original author(s) and the source, provide a link to the Creative Commons license, and indicate if changes were made.

\section{REFERENCES}

1. Dry Eye Workshop (DEWS) Committee. 2007 Report of the International Dry Eye Workshop (DEWS. Ocul Surf. 2007;5(2):65-204.

2. Baudouin C. The pathology of dry eye. Surv Ophthalmol. 2001;45(Suppl 2):S211-20.

3. Yeh S, Song XJ, Farley W, et al. Apoptosis of ocular surface cells in experimentally induced dry eye. Invest Ophthalmol Vis Sci. 2003;44:124-9.

4. Sharma A, Hindman HB. Aging: a predisposition to dry eyes. J Ophthalmol. 2014;2014:781683.

5. Brzhesky BB, Somov EE. Corneal and conjunctival xerosis (diagnosis, clinical features, management). 2nd ed. St. Petersburg: Levsha; 2003. pp 119.

6. Brzheskiy BB, Somov EE. Current treatment of corneal and conjunctival xerosis. Proceedings of the 3rd Russian National School Ophthalmologists. 2004. p. 250-7.

7. Tsubota $\mathrm{K}$, Kawashima $\mathrm{M}$, Inaba $\mathrm{T}$, et al. The antiaging approach for the treatment of dry eye. Cornea. 2012;31(Suppl 1):S3-8.

8. Wakamatsu TH, Dogru M, Tsubota K. Tearful relations: oxidative stress, inflammation and eye diseases. Arq Bras Oftalmol. 2008;71(Suppl 6):72-9.

9. Skulachev VP. Functions of mitochondria: from intracellular power stations to mediators of a senescence program. Cell Mol Life Sci. 2009;66(11-12):1785-93.

10. Korshunov SS, Skulachev VP, Starkov AA. High protonic potential actuates a mechanism of production of reactive oxygen species in mitochondria. FEBS Lett. 1997;416:15-8.

11. Kelso GF, Porteous CM, Coulter CV, et al. Selective targeting of a redox-active ubiquinone to mitochondria within cells: antioxidant and 
antiapoptotic properties. J Biol Chem. 2001;276:4588-96.

12. Antonenko YN, Avetisyan AV, Bakeeva LE, et al. Mitochondria-targeted plastoquinone derivatives as tools to interrupt execution of the aging program. 1. Cationic plastoquinone derivatives: Synthesis and in vitro studies. Biochemistry (Moscow). 2008;73:1273-87.

13. Skulachev VP, Anisimov VN, Antonenko YN, et al. An attempt to prevent senescence: a mitochondrial approach. Biochim Biophys Acta. 2009;1787:437-61.

14. Skulachev MV, Antonenko YN, Anisimov VN, et al. Mitochondrial-targeted plastoquinone derivatives. Effect on senescence and acute age-related pathologies. Curr Drug Targets. 2011;12:800-26.

15. Neroev VV, Archipova MM, Bakeeva LE, et al. Mitochondria-targeted plastoquinone derivatives as tools to interrupt execution of the aging program. 4. Age-related eye disease. SkQ1 returns vision to blind animals. Biochemistry (Moscow). 2008;73:1317-28.

16. Snytnikova OA, Tsentalovich YP, Stefanova NA, et al. The therapeutic effect of mitochondria-targeted antioxidant SkQ1 and Cistanche deserticola is associated with increased levels of tryptophan and kynurenine in the rat lens. Dokl Biochem Biophys. 2012;447:300-3.

17. Rumyantseva YV, Ryabchikova EI, Fursova AZ, Kolosova NG. Ameliorative effects of SkQ1 eye drops on cataractogenesis in senescence-accelerated OXYS rats. Graefes Arch Clin Exp Ophthalmol. 2015;253(2):237-48.

18. Yani EV, Katargina LA, Chesnokova NB, et al. The first experience of using the drug Vizomitin in the treatment of dry eyes. Pract Med (Russ). 2012;4(59):134-7.

19. Wikipedia. Golovin-Sivtsev table 2015. Available at: https://en.wikipedia.org/wiki/Golovin\%E2\%80\% 93Sivtsev_table. Accessed 21 June 2015.
20. Módis L, Szalai E. Dry eye diagnosis and management. Expert Rev Ophthalmol. 2011;6:67-79.

21. Baudouin C, Aragona P, Van Setten G, et al. Diagnosing the severity of dry eye: a clear and practical algorithm. $\mathrm{Br} \mathrm{J}$ Ophthalmol. 2014;98(9):1168-76.

22. Zeev MS, Miller DD, Latkany R. Diagnosis of dry eye disease and emerging technologies. Clin Ophthalmol. 2014;8:581-90.

23. Schein OD, Tielsch JM, Munoz B, Bandeen-Roche K, West S. Relation between signs and symptoms of dry eye in the elderly. A population-based perspective. Ophthalmology. 1997;104:1395-401.

24. Foulks GN. Challenges and pitfalls in clinical trials of treatments for dry eye. Ocul Surf. 2003;1:20-30.

25. Nichols KK, Nichols JJ, Mitchell GL. The lack of association between signs and symptoms in patients with dry eye disease. Cornea. 2004;23:762-70.

26. Sullivan BD, Whitmer D, Nichols KK, et al. An objective approach to dry eye disease severity. Invest Ophthalmol Vis Sci. 2010;51:6125-6.

27. Tutt R, Bradley A, Begley C, Thibos LN. Optical and visual impact of tear break-up in human eyes. Invest Ophthalmol Vis Sci. 2000;41:4117-23.

28. Lemp MA. Advances in understanding and managing dry eye disease. Am J Ophthalmology. 2008;146:350-6.

29. Pflugfelder SC. Antiinflammatory therapy for dry eye. Am J Ophthalmol. 2004;137(2):337-42.

30. Zinovkin RA, Romaschenko VP, Galkin II, et al. Role of mitochondrial reactive oxygen species in age-related inflammatory activation of endothelium. Aging (Albany NY). 2014;6(8):661-74. 